\title{
Cooperative Power and DoT Estimation for a Directive Source
}

\author{
Sina Maleki ${ }^{\dagger}$, Philippe Ciblat ${ }^{\star}$, Symeon $_{\text {Chatzinotas }}^{\dagger}$, Dzevdan Kapetanovic ${ }^{\ddagger}$, and Björn Ottersten ${ }^{\dagger}$ \\ ${ }^{\dagger}$ Interdisciplinary Centre for Security, Trust and Reliability (SnT), \\ University of Luxembourg, Luxembourg, \\ e-mail: \{sina.maleki, symeon.chatzinotas, bjorn.ottersten\}@uni.lu \\ * Telecom ParisTech, Paris, e-mail: philippe.ciblat@enst.fr \\ ${ }_{\ddagger}^{\ddagger}$ Ericsson, Lund, Sweden, e-mail: dzevdan.kapetanovic@ericsson.com
}

\begin{abstract}
Reliable estimation of the source power as well as the direction of transmission (DoT) is required in a large number of applications, e.g. radio environment mapping for cognitive radios, security, system performance and interference monitoring. In this paper, we develop a multi-sensor cooperative estimation algorithm for joint power and DoT estimation of a source with a known location and equipped with a directive antenna pattern. The source signal is assumed to be known, e.g. a training sequence, and the channel is modeled by the free-space path loss. Simulation results show that the developed algorithm can deliver a reliable estimation accuracy.

Index Terms-Direction of transmission (DoT), ML estimation, spectrum cartography, cognitive radio, directive source
\end{abstract}

\section{INTRODUCTION}

Spectrum cartography or radio environment mapping (REM) is considered as an enabling technique for large cognitive radio networks implementation [1], [2]. REM can be used as a database for cognitive radios to allocate the available resources while adhering to the regulatory interference constraints. Several techniques are proposed in the literature in order to obtain REM at a large scale. In [2], the authors exploit the inherent sparsity in the frequency and spatial domain in order to obtain the spectrum map of the sources. Location estimation information is employed in [3] in order to reconstruct the spectrum map. Different parameters of the primary radio network are estimated in [4], and the results are stored in a database for REM. The authors of [5] follow a similar approach by estimation of the primary network parameters as well as deriving the theoretical bounds on the estimation accuracy of the proposed algorithms. The majority of the current research works consider the source to be omni-directional. While this assumption may be valid in the lower part of the spectrum such as the TV whitespace, due to the directive nature of communications in the higher part of the spectrum (e.g. fixed-service terrestrial or satellite links in $18 \mathrm{GHz}$ and above [6]), the developed algorithms may fail to achieve a good accuracy. Therefore, in order to obtain a reliable spectrum map, it is necessary to estimate the direction of transmission (DoT) (i.e., the direction of antenna with the maximum gain) as well as the location and the transmitted power.

It is important to note that DoT estimation is different from the direction of arrival (DoA) estimation which is a well studied technique in the literature [7]-[9], particularly when it comes to near-field effects. To the best of our knowledge except [4] and [5], there are no other work which takes the antenna directivity into account for spectrum cartography. In [4], an extensive set of measurements over different distances and positions is used in order to estimate the direction of transmission. Exhaustive search over multiple dimensions and large number of sensors is performed to estimate the direction of transmission in [5]. Further, the developed techniques only consider the case with Gaussian shaped antenna radiation patterns. Although, the main lobe of the symmetric antennas (e.g. horn antenna) can be modeled approximately as Gaussian, however not all the sensors may be located in the main lobe, and further not all the antenna patterns are symmetric, and thus a more general model need to be considered.

In this paper, a joint power and DoT estimation algorithm is developed which can be applied to any antenna with a known radiation pattern. We particularly consider a case where a few number of sensors are distributed around a transmitter and collects measurement samples. They send the samples to a fusion center (FC) which is responsible to infer them, and estimate the power and DoT of the source. The sensors are assumed to be fixed during the measurements, and their locations are known to the FC. Further, the location and the signal of the transmitter are known to the FC, e.g. a training sequence, (the case with the unknown random signal is considered in [10]). This information can be either obtained through databases with minimal information about the incumbent users, or estimated beforehand [11]. The FC solves a maximum-likelihood (ML) problem in order to estimate the underlying parameters. As shall be shown later, for a given DoT, the power can be estimated by a closed-form solution. This solution is then used by the ML estimator to jointly estimate the power and DoT which maximizes the likelihood function. It is clear that knowing the location, the transmission power, and the DoT, the spectrum map of the source over the considered geographical area can be produced.

The remainder of the paper is organized as follows. The system model and problem formulation is discussed in Section II. We provide the solution to the problem in Section III. The related Cramer-Rao Bound (CRB) is derived in Section IV. Simulation results are plotted in Section V. Finally, we draw 
our conclusions as well as future lines of work in Section VI.

\section{Problem StATEMENT}

We consider a source which employs a directive antenna with a known radiation pattern. The transmission occurs in a deterministic but unknown direction. The direction of transmission (DoT) is denoted by angle $\phi$ towards a specific reference line and represents the direction of the main lobe. We denote $P_{s}$ as the unknown source transmission power, and $G$ as the antenna gain. We denote by $M>1$ the number of sensors which are located at different angles towards the reference line denoted by $\theta_{i}, i=1, \cdots, M$. The sensors observations are assumed to be independent. We consider a scenario where the sensors are aware of their own locations (and thus the angles $\theta_{i}, i=1, \cdots, M$ ) as well as the location of the other sensors and the source. The sensors send their observations to the FC. The goal of the FC is to estimate $P_{s}$ and $\phi$. Further, we assume that the sensors and the source are fixed during the estimation period. Fig. 1 depicts the setup of the sensors, as well as the cooperative estimation configuration of the sensors and the FC.
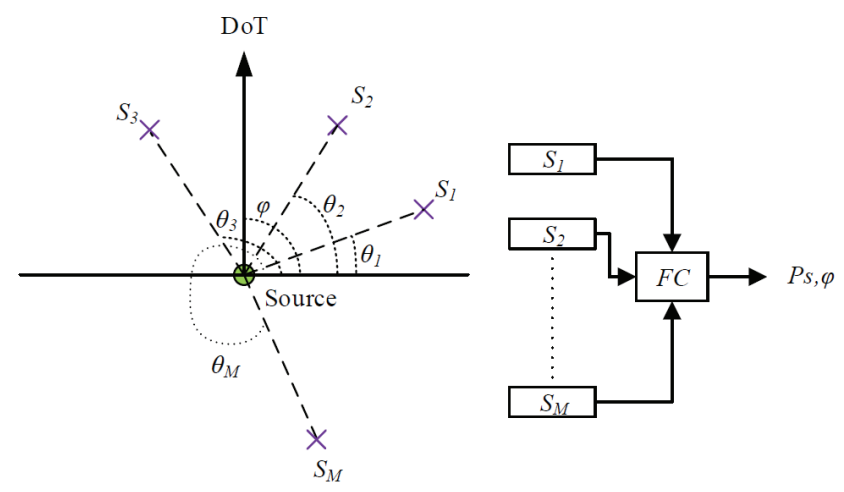

Fig. 1: Schematic configuration of the considered system model.

Denoting $x_{i}[n], i=1, \cdots, M$ to be the received signal at time $n$ and sensor $i$, we have

$$
x_{i}[n]=\sqrt{P_{s} G\left(\phi, \theta_{i}\right) h\left(d_{i}\right)} s[n]+w_{i}[n],
$$

where

- $h\left(d_{i}\right)$ is the path-loss gain,

- $G\left(\phi, \theta_{i}\right)$ is the antenna gain in the direction of sensor $i$,

- $s[n]$ is the known transmitted signal (e.g. the training sequence of a communication system) with $E\left[s^{2}[n]\right]=$ 1 , where $E[\cdot]$ denotes the expectation,

- and $w[n]$ is the i.i.d. additive-white-Gaussian-noise (AWGN) with zero-mean and variance $\sigma_{w}^{2}$.

The path-loss gain is obtained by $h\left(d_{i}\right)=\left(4 \pi d_{i} / \lambda\right)^{-\gamma}$, where $\lambda$ is the source signal wavelength, and $\gamma$ is the path-loss exponent. Note that this channel model does not represent the instantaneous channel variations in wireless communications, however provides a good approximation of attenuation. For sake of simplicity, we consider real-valued signals. Based on the defined system model, $x_{i}[n]$ is an i.i.d. real-valued random Gaussian variable with mean value of $\sqrt{P_{s} G\left(\phi, \theta_{i}\right) h\left(d_{i}\right)} s[n]$ and variance $\sigma_{w}^{2}$. Therefore, the probability density function (pdf) of the received signal at sensor $i$ and time $n$ denoted by $P\left(x_{i}[n]\right)$ becomes

$$
P\left(x_{i}[n]\right)=\frac{1}{\sqrt{2 \pi \sigma_{w}^{2}}} e^{-\frac{\left(x_{i}[n]-\sqrt{P_{s} G\left(\phi, \theta_{i}\right) h\left(d_{i}\right)} s[n]\right)^{2}}{2 \sigma_{w}^{2}}},
$$

where $e$ denotes the exponential function. Before going through the underlying estimation algorithm in Section III, in the following proposition, we provide the identifiability conditions for the data model in (1), which is proved in [10]. Recall that a model parameter in (1) is identifiable if for the error and noise free scenarios, from $P_{s}^{t} G\left(\phi^{t}, \theta_{i}\right)=P_{s} G\left(\phi, \theta_{i}\right)$, we obtain $P_{s}=P_{s}^{t}$, and $\phi=\phi^{t}$, where $\phi^{t}$ and $\phi$ denote the true and estimated DoT, respectively, and $P_{s}^{t}$ and $P_{s}$ denote the true and estimated transmission power.

Proposition 1. The model in (1) is identifiable, if the following conditions are satisfied,

1) $\forall \phi \neq \phi^{t}: \exists \theta_{i}: G\left(\phi, \theta_{i}\right) \neq G\left(\phi^{t}, \theta_{i}\right)$.

2) $\forall \Delta \neq 1$ and $\phi \neq \phi^{t}: \exists \theta_{i}: G\left(\phi, \theta_{i}\right) \neq \frac{1}{\Delta} G\left(\phi^{t}, \theta_{i}\right)$, where $\Delta=\frac{P_{s}}{P_{s}^{t}}$.

The first condition means that there is always at least one sensor which observes a different gain when the DoT changes, and in the second condition, there is always at least one sensor which its received gain does not change in the reverse proportion of the change in the power. Meanwhile, for symmetric antenna patterned sources, we obtain the following proposition which is proved in [10],

Proposition 2: If the source is equipped with a non-linear symmetric antenna gain pattern which is a one-to-one nonlinear decreasing function over $\left|\phi-\theta_{i}\right| \in[0, \omega]$, the model parameters are identifiable if $\theta_{i}=(i-1) \frac{2 \pi}{M}, i=1, \cdots, M$, with $M>\frac{2 \pi}{\omega}$, and $\omega \leq \pi$.

\section{MAXImum-Likelihood Estimation Algorithm}

As mentioned before, all the sensors send their observations $x_{i}[n]$ s sequentially to the FC. The FC then estimates the power and the DoT of the source using maximum likelihood (ML) estimation. Denoting $N$ to be total number of samples, the joint likelihood function denoted by $L$ is obtained as follows

$$
L\left(\phi, P_{s}\right)=\prod_{n=1}^{N} \prod_{i=1}^{M} P\left(x_{i}[n]\right)
$$

and after some simplifications, the log-likelihood (LL) function becomes

$$
\begin{aligned}
& L L\left(\phi, P_{s}\right)=M N \log \frac{1}{\sqrt{2 \pi \sigma_{w}^{2}}} \\
& -\frac{1}{2 \sigma_{w}^{2}}\left[\sum_{i=1}^{M} \sum_{n=1}^{N}\left(x_{i}[n]-\sqrt{P_{s} G\left(\phi, \theta_{i}\right) h\left(d_{i}\right)} s[n]\right)^{2}\right] .
\end{aligned}
$$

Since $M N \log \frac{1}{\sqrt{2 \pi \sigma_{w}^{2}}}$ and $\frac{1}{2 \sigma_{w}^{2}}$ do not depend on $P_{s}$ or $\phi$, for estimation purposes we consider a reduced version of LL 
function in (4) denoted by $L L_{r}$ as follows

$L L_{r}\left(\phi, P_{s}\right)=-\left[\sum_{i=1}^{M} \sum_{n=1}^{N}\left(x_{i}[n]-\sqrt{P_{s} G\left(\phi, \theta_{i}\right) h\left(d_{i}\right)} s[n]\right)^{2}\right]$.

In order to estimate $P_{s}$ and $\phi$, we consider the following ML estimation problem defined as

$$
\begin{aligned}
\max _{P_{s}, \phi} & L L_{r}\left(\phi, P_{s}\right) \\
& \text { s.t. } P_{s} \geq 0,0^{\circ} \leq \phi<360^{\circ},
\end{aligned}
$$

where $L L_{r}\left(\phi, P_{s}\right)$ is obtained from (5). To solve (6), first we assume that the $\phi$ is given and find the optimal $P_{s}$, and then we insert the optimal $P_{s}$ in (6) to find the optimal $\phi$. As shall be shown later, for a given $\phi$ denoted by $\phi_{g}$, there is a unique $P_{s}$ which maximizes (5). For $\phi_{g}$, (6) becomes

$$
\begin{aligned}
\max _{P_{s}} & -\left[\sum_{i=1}^{M} \sum_{n=1}^{N}\left(x_{i}[n]-\sqrt{P_{s} G\left(\phi_{g}, \theta_{i}\right) h\left(d_{i}\right)} s[n]\right)^{2}\right] \\
\text { s.t. } P_{s} \geq 0 . &
\end{aligned}
$$

We obtain the following theorem which provides the closed form solution of Eq. (7) denoted by $P_{s}^{*}\left(\phi_{g}\right)$.

Theorem 1: The optimal solution of Eq. (7) is obtained by

- If $\sum_{i=1}^{M} R_{i} \sqrt{G\left(\phi_{g}, \theta_{i}\right) h\left(d_{i}\right)}>0$, then

$$
P_{s}^{*}\left(\phi_{g}\right)=\left(\frac{\sum_{i=1}^{M} R_{i} \sqrt{G\left(\phi_{g}, \theta_{i}\right) h\left(d_{i}\right)}}{N \sum_{i=1}^{M} G\left(\phi_{g}, \theta_{i}\right) h\left(d_{i}\right)}\right)^{2}
$$

where $R_{i}=\sum_{n=1}^{N} x_{i}[n] s[n]$.

- If $\sum_{i=1}^{M} R_{i} \sqrt{G\left(\phi_{g}, \theta_{i}\right) h\left(d_{i}\right)} \leq 0$, then

$$
P_{s}^{*}\left(\phi_{g}\right)=0 \text {. }
$$

Proof. The proof is provided in Appendix A.

We can now rewrite Eq. (6) as follows

$$
\begin{aligned}
\max _{\phi} & L L_{r}\left(\phi, P_{s}^{*}(\phi)\right) \\
& \text { s.t. } 0^{\circ} \leq \phi<360^{\circ},
\end{aligned}
$$

where $P_{s}^{*}(\phi)$ is the optimal $P_{s}$ coming from Theorem 1. After some simple algebraic simplifications, we obtain the following Theorem.

Theorem 2: The function to be maximized in Eq. (9) can be rewritten as follows

$$
\begin{aligned}
\max _{\phi} & U\left(\sum_{i=1}^{M} R_{i} \sqrt{G\left(\phi, \theta_{i}\right) h\left(d_{i}\right)}\right) \\
\times & \frac{\left(\sum_{i=1}^{M} R_{i} \sqrt{G\left(\phi, \theta_{i}\right) h\left(d_{i}\right)}\right)^{2}}{\sum_{i=1}^{M} G\left(\phi, \theta_{i}\right) h\left(d_{i}\right)}
\end{aligned}
$$

where $U(\bullet)$ is the Heavyside function, i.e., $U(x)=1$ if $x \geq 0$ and $U(x)=0$ otherwise. Therefore, in order to solve (6) optimally, a line search over $\phi$ as in Theorem 2 is sufficient to find the optimal $\phi$, and consequently the optimal $P_{s}$ using the expression in Theorem 1.

\section{CRB ANALYSIS}

In order to compare the performance of the developed technique, here we obtain the Cramer-Rao-Bound (CRB) of the estimation technique developed in this paper. The CRB provides a lower-bound on the mean-square-error (MSE) of an unbiased estimator and thus $\operatorname{MSE}\left(P_{s}, \phi\right) \geq \operatorname{CRB}\left(P_{s}, \phi\right)$ [12].

Assuming that $L L\left(P_{s}, \phi\right)$ satisfies the regulatory conditions, after algebraic manipulations presented in Appendix $\mathrm{B}$, we obtain the following Theorem which calculates $\operatorname{CRB}\left(P_{s}, \phi\right)=\operatorname{CRB}\left(P_{s}\right)+\operatorname{CRB}(\phi)$.

Theorem 3: The $\operatorname{CRB}\left(P_{s}, \phi\right)$ for deterministic signal is given by

$$
\begin{aligned}
\operatorname{CRB}\left(P_{s}, \phi\right)= & \frac{4 P_{s} \sigma_{w}^{2}}{N \sum_{i=1}^{M} G\left(\phi, \theta_{i}\right) h\left(d_{i}\right)} \\
& +\frac{4 \sigma_{w}^{2}}{N P_{s} \sum_{i=1}^{M} h\left(d_{i}\right) \frac{G^{\prime 2}\left(\phi, \theta_{i}\right)}{G\left(\phi, \theta_{i}\right)}},
\end{aligned}
$$

where $G^{\prime}\left(\phi, \theta_{i}\right)=\frac{\partial G\left(\phi, \theta_{i}\right)}{\partial \phi}$, and individual $\operatorname{CRB}\left(P_{s}\right)$ and $\operatorname{CRB}(\phi)$ are

$$
\begin{gathered}
\operatorname{CRB}\left(P_{s}\right)=\frac{4 P_{s} \sigma_{w}^{2}}{N \sum_{i=1}^{M} G\left(\phi, \theta_{i}\right) h\left(d_{i}\right)}, \\
\operatorname{CRB}(\phi)=\frac{4 \sigma_{w}^{2}}{N P_{s} \sum_{i=1}^{M} h\left(d_{i}\right) \frac{G^{\prime 2}\left(\phi, \theta_{i}\right)}{G\left(\phi, \theta_{i}\right)}} .
\end{gathered}
$$

Note that the calculation of individual CRBs is merely provided to gain more insights. Otherwise, as the estimation is jointly performed over $P_{s}$ and $\phi$, the individual CRBs can not be a good benchmark for comparison. From (10), it is clear that increasing the noise power, increases the total CRB, but the effect of $P_{s}$ on the total CRB is not exactly clear. Increasing $P_{s}$ increases the $\operatorname{CRB}\left(P_{s}\right)$ but reduces the $\operatorname{CRB}(\phi)$. Additionally, increasing the number of samples reduces the total CRB linearly and thus the expected MSE. Furthermore, we can see that as the number of sensors increases, the CRB decreases but its effect is not linearly scaled as is the case for the number of samples $N$. Finally, it is clear that as the distance of the sensors to the source increases, CRB increases.

\section{Simulation Results}

In this section, we evaluate the performance of the developed algorithm in terms of the normalized mean square error (NMSE). Without loss of generality, we assume that the sensors are located with the same distance to the source, and further they are equally distanced from each other over a circle with radius $d$, and centered on the source, and $s=1$. In all figures we assume the antenna gain is modeled by

$G\left(\phi, \theta_{i}\right)= \begin{cases}100 \exp \left(-\left|\phi-\theta_{i}\right|\right) & \text { if } 0^{\circ} \leq\left|\phi-\theta_{i}\right| \leq 180^{\circ}, \\ 0 & \text { else, }\end{cases}$

where $|\cdot|$ denotes the absolute value. Note that this model is an approximation of a symmetric antenna gain pattern, e.g. Horn antennas, and can provide good insights in the behavior of the proposed estimation algorithm. Each result is obtained by 
averaging over 1000 runs. Further, in order to make sure the simulation setups are identifiable, we follow the identifiability analysis provided in Proposition 2.

The convergence of the proposed technique with the number of samples is evaluated in Fig. 2 for a case of 3 sensors. In this figure, the NMSE versus the number of samples is depicted for both $P_{s}$ and $\phi$. It is clear that the proposed ML algorithm converges after few samples and estimation error is very low.

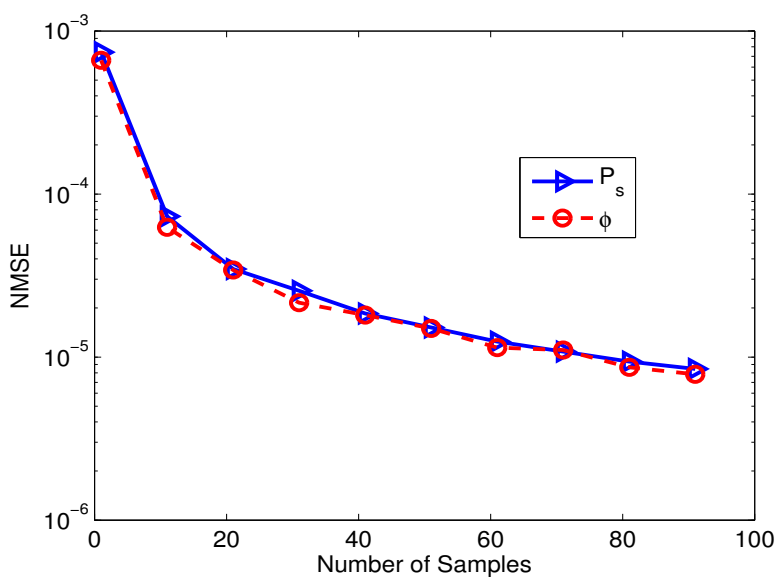

Fig. 2: Normalized mean square error (NMSE) with number of samples, $\phi=60^{\circ}, d_{1}=d_{2}=d_{3}=100 \mathrm{~m}, \theta_{1}=0^{\circ}$, $\theta_{2}=120^{\circ}, \theta_{3}=240^{\circ}, P_{s}=0 \mathrm{dBW}$, and $\sigma_{w}^{2}=-136 \mathrm{dBW}$.

We follow the simulations in Fig. 3 by comparing $\operatorname{NMSE}\left(P_{s}, \phi\right)=\operatorname{NMSE}\left(P_{s}\right)+\operatorname{NMSE}(\phi)$, with $\operatorname{NCRB}\left(P_{s}, \phi\right)=\operatorname{NCRB}\left(P_{s}\right)+\operatorname{NCRB}(\phi)$ as the number of samples increases. The simulation setup remains the same as in Fig. 2. We can see that the estimation accuracy of the proposed algorithm is close to the CRB.

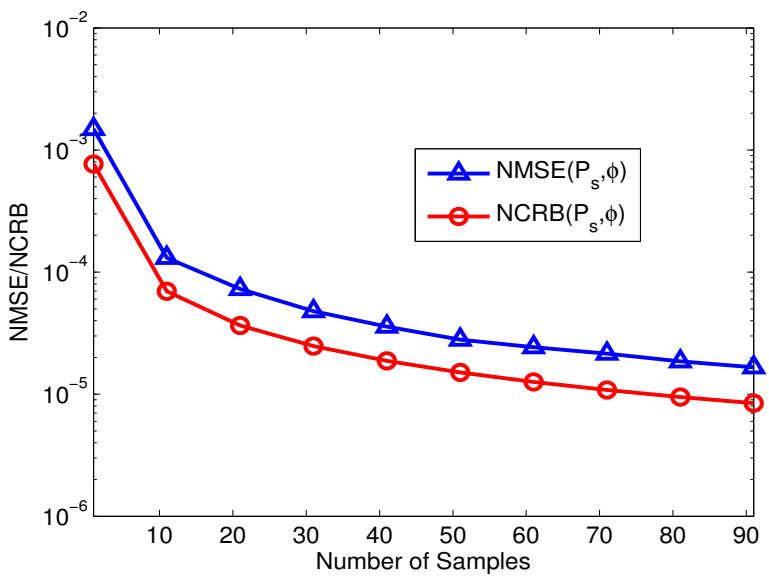

Fig. 3: Normalized mean square error (NMSE) versus normalized CRB (NCRB), $\phi=60^{\circ}, d_{1}=d_{2}=d_{3}=100 \mathrm{~m}$, $\theta_{1}=0^{\circ}, \theta_{2}=120^{\circ}, \theta_{3}=240^{\circ}, P_{s}=0 \mathrm{dBW}$, and $\sigma_{w}^{2}=-136 \mathrm{dBW}$.
In Figures 4 and 5, for the same antenna pattern as in Fig. 2, we study the estimation accuracy as it changes with the distance of the sensors to the source. From these figures, we can see that the cooperative estimation technique developed in this paper can reliably estimate $P_{s}$ and $\phi$, and as the number of sensors increases, the estimation accuracy improves. Further, we can see that the distance to the source has a relatively significant impact on the estimation accuracy. In these two figures, the angular position of the sensors for the case with 3 sensors is the same as Fig. 2, while for the case with 4 sensors $\theta_{1}=0^{\circ}, \theta_{2}=90^{\circ}, \theta_{3}=180^{\circ}, \theta_{4}=270^{\circ}$.

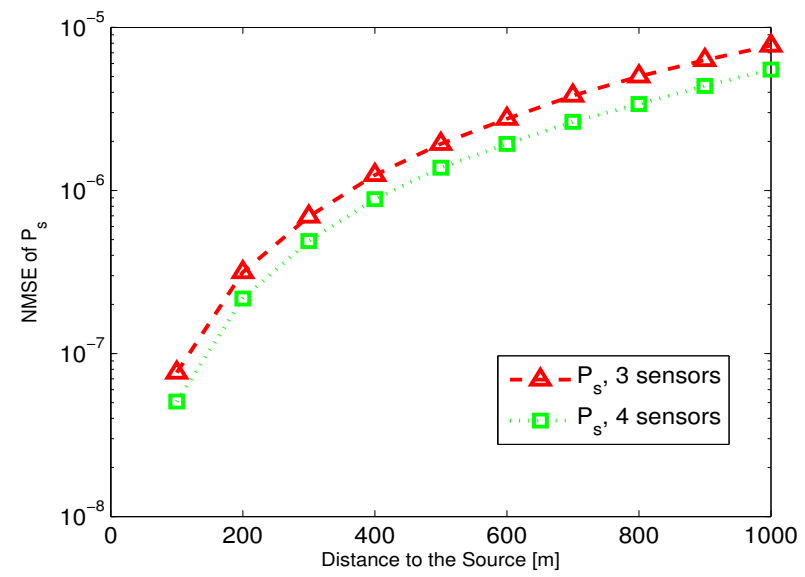

Fig. 4: Normalized mean square error of $P_{s}$ estimation, $\phi=$ $60^{\circ}, d_{i}=100-1000 \mathrm{~m}, P_{s}=0 \mathrm{dBW}, \sigma_{w}^{2}=-136 \mathrm{dBW}$.

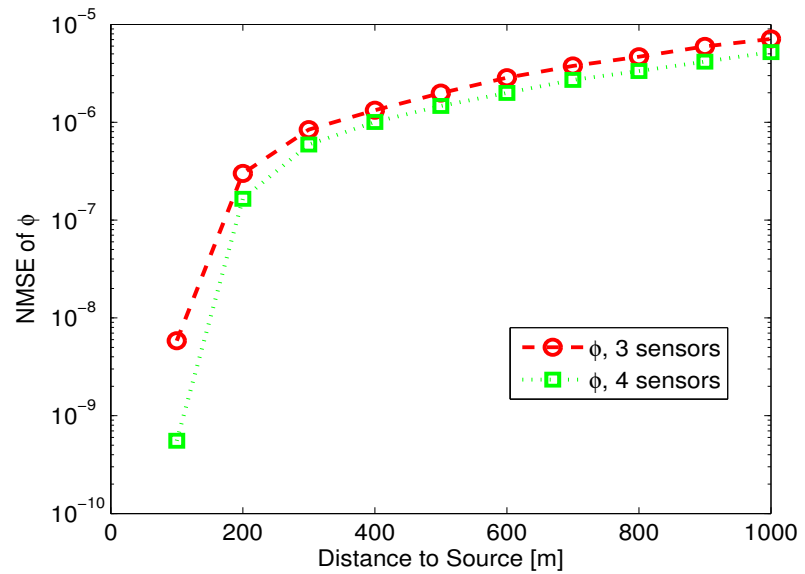

Fig. 5: Normalized mean square error with $\phi$ estimation, $\phi=$ $60^{\circ}, d_{i}=100-1000 \mathrm{~m}, P_{s}=0 \mathrm{dBW}, \sigma_{w}^{2}=-136 \mathrm{dBW}$.

\section{CONCLUSION}

In this paper, we developed a cooperative estimation algorithm to jointly estimate the source power and DoT. Assuming a known signal model, a closed form solution for the source power estimation was provided for a given DoT. Afterward, DoT can be easily estimated by a line search over $\phi$. Several 
simulations results were provided in support of the proposed algorithm. It was shown that the algorithm can reliably estimate the desired parameters and performs close to the CRB. In this work, we assumed a simplistic channel model by only taking path loss into account. Considering more realistic signal and channel models are subjects of the future work.

\section{APPENDIX A \\ Proof OF THEOREM 1}

In order to find the maximum of $P_{s} \mapsto L L_{r}\left(P_{s}, \phi_{g}\right)$, we would like to analyze the shape of the function. To do that, we will calculate its derivative function. For any $P_{s} \neq 0$, we easily get

$$
\begin{aligned}
\frac{\partial L L_{r}\left(P_{s}, \phi_{g}\right)}{\partial P_{s}} & =\frac{1}{\sqrt{P_{s}}} \sum_{i=1}^{M} R_{i} \sqrt{G\left(\phi_{g}, \theta_{i}\right) h\left(d_{i}\right)} \\
& -N \sum_{i=1}^{M} G\left(\phi_{g}, \theta_{i}\right) h\left(d_{i}\right) .
\end{aligned}
$$

- If $\sum_{i=1}^{M} R_{i} \sqrt{G\left(\phi_{g}, \theta_{i}\right) h\left(d_{i}\right)}>0$, then the derivative function is positive as $P_{s} \rightarrow 0$. And thus the function $L L_{r}\left(\bullet, \phi_{g}\right)$ increases with $P_{s}$ until the point $P_{s}^{*}$ such that

$$
\frac{1}{\sqrt{P_{s}^{*}}} \sum_{i=1}^{M} R_{i} \sqrt{G\left(\phi_{g}, \theta_{i}\right) h\left(d_{i}\right)}=N \sum_{i=1}^{M} G\left(\phi_{g}, \theta_{i}\right) h\left(d_{i}\right) .
$$

Beyond the point $P_{s}^{*}$, the derivative function becomes negative and the function $L L_{r}\left(\bullet, \phi_{g}\right)$ decreases. Therefore the optimal point is $P_{s}^{*}$ and so we get Eq. (8).

- If $\sum_{i=1}^{M} R_{i} \sqrt{G\left(\phi_{g}, \theta_{i}\right) h\left(d_{i}\right)} \leq 0$, then the derivative function is always negative and so the function $L L_{r}\left(\bullet, \phi_{g}\right)$ is monotonic decreasing in $P_{s}$. Therefore the optimal point is zero.

\section{APPENDIX B}

\section{Proof OF THEOREM 3}

We recall that the $\mathrm{CRB}$ for parameters $\left[P_{s}, \phi\right]$ is the trace of the inverse of the Fisher Information Matrix F ( [12]) defined as

$$
\mathbf{F}=\mathbb{E}\left[\begin{array}{ll}
\frac{\partial L L}{\partial P_{s}} \frac{\partial L L}{\partial P_{s}} & \frac{\partial L L}{\partial P_{s}} \frac{\partial L L}{\partial \phi} \\
\frac{\partial L L}{\partial \phi} \frac{\partial L L}{\partial P_{s}} & \frac{\partial L L}{\partial \phi} \frac{\partial L L}{\partial \phi}
\end{array}\right],
$$

where $L L\left(P_{s}, \phi\right)$ is given by (4). After some calculations we can derive each term of the $\mathbf{F}$ matrix as follows,

$$
\begin{array}{r}
\mathbb{E}\left(\frac{\partial L L}{\partial P_{s}} \frac{\partial L L}{\partial P_{s}}\right)=\frac{N \sum_{i=1}^{M} G\left(\phi, \theta_{i}\right) h\left(d_{i}\right)}{4 P_{s} \sigma_{w}^{2}}, \\
\mathbb{E}\left(\frac{\partial L L}{\partial \phi} \frac{\partial L L}{\partial \phi}\right)=\frac{N P_{s} \sum_{i=1}^{M} h\left(d_{i}\right) \frac{G^{\prime 2}\left(\phi, \theta_{i}\right)}{G\left(\phi, \theta_{i}\right)}}{4 \sigma_{w}^{2}},
\end{array}
$$

with $G^{\prime}\left(\phi, \theta_{i}\right)=\frac{\partial G\left(\phi, \theta_{i}\right)}{\partial \phi}$, and

$$
\mathbb{E}\left(\frac{\partial L L}{\partial P_{s}} \frac{\partial L L}{\partial \phi}\right)=\mathbb{E}\left(\frac{\partial L L}{\partial \phi} \frac{\partial L L}{\partial P_{s}}\right)=0 .
$$

This way, the inverse of $\mathbf{F}$ denoted by $\mathbf{F}^{-1}$ becomes

$$
\mathbf{F}^{-1}=\left[\begin{array}{cc}
\frac{4 P_{s} \sigma_{w}^{2}}{N \sum_{i=1}^{M} G\left(\phi, \theta_{i}\right) h\left(d_{i}\right)} & 0 \\
0 & \frac{4 \sigma_{w}^{2}}{N P_{s} \sum_{i=1}^{M} h\left(d_{i}\right) \frac{G^{\prime 2}\left(\phi, \theta_{i}\right)}{G\left(\phi, \theta_{i}\right)}}
\end{array}\right]_{(18)}
$$

and thus we obtain

$$
\begin{aligned}
\operatorname{CRB}\left(P_{s}, \phi\right)= & \operatorname{trace}\left(\mathbf{F}^{-1}\right)=\frac{4 P_{s} \sigma_{w}^{2}}{N \sum_{i=1}^{M} G\left(\phi, \theta_{i}\right) h\left(d_{i}\right)} \\
& +\frac{4 \sigma_{w}^{2}}{N P_{s} \sum_{i=1}^{M} h\left(d_{i}\right) \frac{G^{\prime 2}\left(\phi, \theta_{i}\right)}{G\left(\phi, \theta_{i}\right)}},
\end{aligned}
$$

and

$$
\begin{gathered}
\operatorname{CRB}\left(P_{s}\right)=\frac{4 P_{s} \sigma_{w}^{2}}{N \sum_{i=1}^{M} G\left(\phi, \theta_{i}\right) h\left(d_{i}\right)}, \\
\operatorname{CRB}(\phi)=\frac{4 \sigma_{w}^{2}}{N P_{s} \sum_{i=1}^{M} h\left(d_{i}\right) \frac{G^{\prime 2}\left(\phi, \theta_{i}\right)}{G\left(\phi, \theta_{i}\right)}},
\end{gathered}
$$

which concludes our proof.

\section{ACKNOWLEDGMENT}

This work is partially supported by the National Research Fund, Luxembourg under the projects SeMIGod, and SATSENT as well as European project H2020 SANSA.

\section{REFERENCES}

[1] H.B. Yilmaz, T. Tugcu, F. Alagoz, S. Bayhan, "Radio environment map as enabler for practical cognitive radio networks," IEEE Communications Magazine, vol.51, no.12, pp.162-169, December 2013.

[2] J.A. Bazerque, G.B. Giannakis, "Distributed Spectrum Sensing for Cognitive Radio Networks by Exploiting Sparsity," IEEE Transactions on Signal Processing, vol.58, no.3, pp.1847-1862, March 2010.

[3] H.B. Yilmaz, T. Tugcu "Location estimation-based radio environment map construction in fading channels", Wirel. Commun. Mob. Comput., vol.15, pp.561-570, 2015.

[4] L. Bolea, J. Perez-Romero, R. Agusti, O. Sallent, "Context Discovery Mechanisms for Cognitive Radio," IEEE Vehicular Technology Conference (VTC Spring), pp.1-5, May 2011.

[5] R.K. Martin, R. Thomas, "Algorithms and bounds for estimating location, directionality, and environmental parameters of primary spectrum users," IEEE Transactions on Wireless Communications, vol.8, no.11, pp.56925701, November 2009.

[6] S. Maleki, S. Chatzinotas, B. Evans, K. Liolis, J. Grotz, A. Vanelli-Coralli, N. Chuberre, "Cognitive Spectrum Utilization in Ka Band Multibeam Satellite Communications", to appear in IEEE Communications Magazine.

[7] H. Krim, M. Viberg, "Two decades of array signal processing research: the parametric approach," IEEE Signal Processing Magazine, vol.13, no.4, pp.67-94, July 1996.

[8] M. Viberg, and B. Ottersten, "Sensor array processing based on subspace fitting," IEEE Transactions on Signal Processing, vol.39, no.5, pp.11101121, May 1991.

[9] P. Stoica, A. Nehorai "MUSIC, maximum likelihood, and Cramer-Rao bound," IEEE Transactions on Acoustics, Speech and Signal Processing, vol.37, no.5, pp.720-741, May 1989.

[10] S. Maleki, P. Ciblat, B. Shankar M.R., S. Chatzinotas, B. Ottersten, "Power and direction of transmission estimation for a directive source: identifiability analysis and estimation algorithm", submitted to ICASSP 2016.

[11] Y-D. Huang, M. Barkat, "Near-field multiple source localization by passive sensor array," IEEE Transactions on Antennas and Propagation, vol.39, no.7, pp.968-975, July 1991.

[12] S.M. Kay, "Fundamentals of statistical signal processing, volume I: Estimation theory.", Prentice Hall PTR, 1993. 